\title{
Caracterização da utilização de painel de mensagem variável nos pontos de parada de ônibus de São Paulo
}

\author{
Patricia Sauri Lavieri ${ }^{1}$, Orlando Strambi ${ }^{2}$, Renato Arbex ${ }^{3}$ e Elaine Cristina Schneider de Carvalho ${ }^{4}$
}

\begin{abstract}
Resumo: O artigo analisa a qualidade das informações sobre previsões de chegada de veículos a pontos de ônibus (disponibilizadas nas paradas através de painéis de mensagem variável), bem como os determinantes do uso destas informações por parte dos passageiros. Os dados foram coletados em duas paradas no corredor Campo Limpo/Rebouças/Centro em São Paulo. Foram realizados dois tipos de coletas de dados, em diferentes períodos do dia. Primeiro, foram registradas as previsões mostradas nos painéis e o momento das chegadas dos veículos aos pontos, o que permitiu avaliar a qualidade da informação apresentada. Segundo, foram realizadas entrevistas com os usuários sobre o uso dos painéis. Os resultados indicam que a qualidade (objetiva) das informações disponibilizadas, assim como a percepção dos usuários (subjetiva) da qualidade podem ser consideradas boas. A estimação de um modelo logit binomial permitiu verificar quais características socioeconômicas e de utilização do sistema por parte dos entrevistados influenciam a probabilidade de uso da informação do painel. Foi identificada maior probabilidade de uso do painel para os usuários abaixo de 60 anos e/ou com maior grau de instrução. Também se observa maior uso do painel entre os usuários frequentes e aqueles que podem ser atendidos por mais de uma linha e, portanto, usam as previsões de chegadas para decidir sobre o ônibus a tomar.
\end{abstract}

Palavras-chave: Transporte público, Informação ao usuário, PMV - Painel de Mensagem Variável.

Abstract: The paper analyzes the quality of bus arrival predictions displayed in VMS - Variable Message Signs - in stops, as well as the factors influencing the use of this information by passengers. Two types of data were collected in two stops of a high frequency bus corridor in São Paulo. First, predictions displayed in panels and the effective vehicle arrivals at stops were recorded, which allowed the analysis of the quality of the information provided. Second, interviews were conducted with bus users about their use of the VMSs. Results indicate that the (objective) quality of the information, as well as the quality perceived by users can be considered good. Estimation of a binomial logit model allowed the identification of the importance of user socioeconomic and system utilization characteristics on the likelihood of using the information in the display. Users under 60 or having higher instruction levels have greater probability of using the VMS, as well as individuals using bus service more frequently and those served by more than one line, who can use the information to decide which bus to board.

Keywords: Public transport, User information, Variable Message Sign.

\section{INTRODUÇÃO}

Em São Paulo são transportados por ônibus diariamente cerca de 8 milhões de passageiros, correspondendo a dois terços do total de viagens diárias por transporte público na cidade. Mais de 4 mil quilômetros de vias fazem parte da rede viária utilizada pelos ônibus, passando por mais de 17 mil paradas. No primeiro semestre de 2013, apenas uma parte das vias utilizadas possuía faixas exclusivas para circulação de ônibus - 10 corredores com um total de $126 \mathrm{~km}$ de extensão (valor significativamente ampliado ao longo do último ano) - e pontos de parada equipados com Painéis de Mensagem Variável (Whately, 2012). O controle da operação do sistema de transporte público por ônibus é feito pela São Paulo Transporte (SPTrans), que é responsável pela definição do itinerário das linhas, pela inclusão e supressão de serviços, pela

\footnotetext{
1 Patricia Sauri Lavieri, Escola Politécnica da Universidade de São Paulo; University of Texas at Austi. (laviepa@gmail.com)

2 Orlando Strambi, Escola Politécnica da Universidade de São Paulo. (ostrambi@usp.br)

${ }^{3}$ Renato Arbex, Escola Politécnica da Universidade de São Paulo. (renatoarbex@usp.br)

${ }^{4}$ Elaine Cristina Schneider de Carvalho, Escola Politécnica da

Universidade de São Paulo; Urban Planning Group, Eindhoven University of Technology. (lane_schneider@hotmail.com)

Manuscrito recebido em 22/08/2014 e aprovado para publicação em 19/12/2014.

Este artigo é parte de TRANSPORTES v. 23, n. 1, 2015. ISSN: 2237-1346

(online). DOI:10.4237/transportes.v23i1.836.
}

alteração dos pontos de parada e pelo provimento de informação ao usuário (Ferreira, 2012).

No transporte público por ônibus, os horários de passagem dos veículos pelos pontos de parada dependem tanto da frequência e regularidade do serviço quanto dos congestionamentos na rede viária. Tais congestionamentos aumentam a variabilidade dos tempos de viagem dos veículos, afetando, por consequência, os tempos de espera experimentados pelos usuários nos pontos. Uma forma de auxiliar os passageiros a lidar com a variabilidade dos tempos do transporte público é a divulgação, em tempo real, dos horários previstos para passagem das linhas, através de Painéis de Mensagem Variável (PMV) instalados nos pontos de parada. Na época em que foi realizada esta pesquisa, São Paulo contava com cinco corredores de ônibus (com 160 paradas, no total) equipados com PMV; desde 2012 também são disponibilizadas, pela internet, informações em tempo real sobre a localização dos ônibus nos corredores (Ferreira, 2012), de forma que os usuários possam utilizá-las tanto durante, quanto antes do início de suas viagens.

Efeitos importantes do uso de painéis pelos usuários são identificados por Dziekan e Kottenhoff (2007): diminuição do tempo de espera percebido; redução da incerteza e maior sensação de segurança; aumento da diposição a pagar pelo serviço; melhor uso do tempo de espera e de viagem; efeitos na escolha do modo; maior satisfação e melhor imagem do serviço. Além desses efeitos, Larsen e Sunde (2008) exploram o uso da informação pelo passa- 
geiro para a escolha da linha a ser utilizada quando existe mais de uma possibilidade, aspecto importante ao se modelar a alocação de passageiros às linhas.

A utilização da informação pelo usuário dependerá da ponderação que este fizer quanto aos custos e benefícios envolvidos neste processo, por exemplo, os custos monetários, o investimento de tempo, o esforço ou a atenção necessários em troca da aquisição da informação (Chorus et al., 2006a). Assim, como exposto em Larsen e Sunde (2008), pode ser racional a escolha de permanecer em estado de ignorância quanto às informações do sistema de transporte. Quando o usuário opta por adquirir novas informações, estas são somadas aos seus conhecimentos e experiências prévios, sendo utilizadas de acordo com seu estilo de decisão (Chorus et al., 2006b) e sua atitude em relação ao risco (Bonsall, 2004).

Outro aspecto que influencia a decisão do usuário de adquirir e utilizar as informações é sua confiabilidade (tanto a objetiva quanto a percebida pelo usuário) que, por sua vez, depende da acurácia do sistema. Ben-Elia et al. (2013) apontam que informações imprecisas fornecidas pelo sistema podem agravar o risco percebido pelos usuários. Assim, quanto menor a acurácia do sistema de informações, menores tendem a ser as taxas de utilização destas e maior é a chance de se adotar um comportamento de aversão ao risco, ou seja, a adoção da alternativa mais segura (como, por exemplo, utilizar o primeiro ônibus que passar no ponto). De acordo com Ettema e Timmermans (2006), a experiência permite aos indivíduos conhecerem e avaliarem a confiabilidade das previsões, de modo que há uma distinção entre a previsão e a percepção que o usuário tem dela.

Um relatório publicado em 2003 pelo Transportation Research Board (TRB), sobre o estado da prática dos sistemas de informação ao usuário de ônibus, reúne estudos e dados de três agências americanas e cinco agências internacionais sobre a reação pública à informação em tempo real da chegada de ônibus. De maneira geral as reações dos usuários observadas foram positivas, porém nenhuma agência reportou um aumento na utilização do ônibus como resultado da implantação do sistema de informação. Passageiros viajando à noite passaram a se sentir mais seguros com a informação de quanto tempo esperariam no ponto de parada.

No caso específico de Londres, foram medidas a acurácia do sistema e a percepção do usuário simultaneamente. Observou-se que o sistema gerava em $75 \%$ dos casos previsões de chegada com erros de mais ou menos 2 minutos e em $96 \%$ dos casos, previsões com erros de mais ou menos 5 minutos. Quanto aos usuários, 90\% deles utilizava os PMV e $65 \%$ acreditava esperar por um período menor quando havia a informação de previsão de chegada (o tempo médio de espera percebido passou de 11,9 para 8,6 minutos). Além disso, $64 \%$ dos passageiros entrevistados acreditava que a confiabilidade do serviço havia aumentado após a implantação do sistema de informação, quando na realidade esta havia diminuído (TRB, 2003).

Do exposto acima, é possível concluir que para se avaliar a relação custo-benefício da instalação de infraestrutura e de provimento de informações ao usuário em tempo real é preciso considerar, além de aspectos econômico-financeiros, os seguintes elementos: a qualidade da informação fornecida; como esta qualidade é percebida pelo usuário; se a informação é utilizada ou não e que características estão associadas a esta utilização.

Assim, os objetivos desta pesquisa são: (1) levantar estimativas da qualidade da informação fornecida nas paradas de ônibus de São Paulo equipadas com PMV e identificar a percepção do usuário quanto a esta qualidade; e (2) identificar o uso e o não uso da informação entre os usuários, associando-o com características do indivíduo e do sistema. Para isso, foram levantados dados em campo sobre a operação do sistema e foram feitas entrevistas com usuários de ônibus em pontos equipados com PMV, na cidade de São Paulo.

A análise dos dados coletados em campo, a fim de atender aos dois objetivos expostos acima, envolveu técnicas de estatística descritiva, a proposição e aplicação de critérios de classificação da informação quanto à sua qualidade, bem como a estimação de modelos de escolha discreta do tipo logit binomial para explicar o uso e o não uso do painel.

Este artigo está estruturado em quatro itens. O primeiro introduziu o contexto no qual o estudo está inserido e apresentou uma breve revisão da literatura sobre a interação entre o indivíduo e a provisão de informação ao usuário do transporte público. Em seguida, o item 2 descreve o processo de coleta de dados para o estudo, e o item 3 caracteriza a amostra obtida e analisa os dados de uso do painel. Por fim, o item 4 apresenta as principais conclusões.

\section{COLETA DE DADOS}

Coletar dados sobre um sistema de elevada dimensão e complexidade, contando com recursos limitados, representa um desafio considerável. Portanto, realizou-se a coleta em apenas dois pontos de parada (Eldorado e Clínicas) de um dos principais corredores de ônibus de São Paulo munidos de PMV, o corredor Campo Limpo/Rebouças/Centro. O objetivo não era cobrir espacialmente o sistema, uma vez que se buscava conhecer o comportamento dos usuários. Embora seja esperado que usuários em diferentes regiões da cidade e sujeitos a diferentes padrões de qualidade das previsões em tempo real possam apresentar diferentes comportamentos em relação aos PMV, é razoável admitir que essas diferenças possam ser atribuídas em grande parte às suas distintas características socioeconômicas e aos seus padrões de uso do sistema. Assim, tais dados foram coletados e utilizados na análise. Cabe destacar que os dois pontos selecionados apresentam alta demanda: um deles está próximo ao principal complexo hospitalar de São Paulo e a uma estação de metrô, e o outro localiza-se próximo a um shopping center importante e a uma estação do trem metropolitano.

Os pontos pesquisados servem tanto aos itinerários do centro para o bairro, quanto no sentido oposto. Os dados foram coletados nos dias 6 e 8 de maio de 2013, segunda e quarta-feira, durante aproximadamente uma hora em três períodos diferentes (7h30-8h30; 12h00-13h00 e 17h30-18h30). Estes períodos foram escolhidos de forma a se ter uma amostra dos horários de pico da manhã e da tarde, e de um horário fora do pico. 
Departamento de Engenharia de Transportes

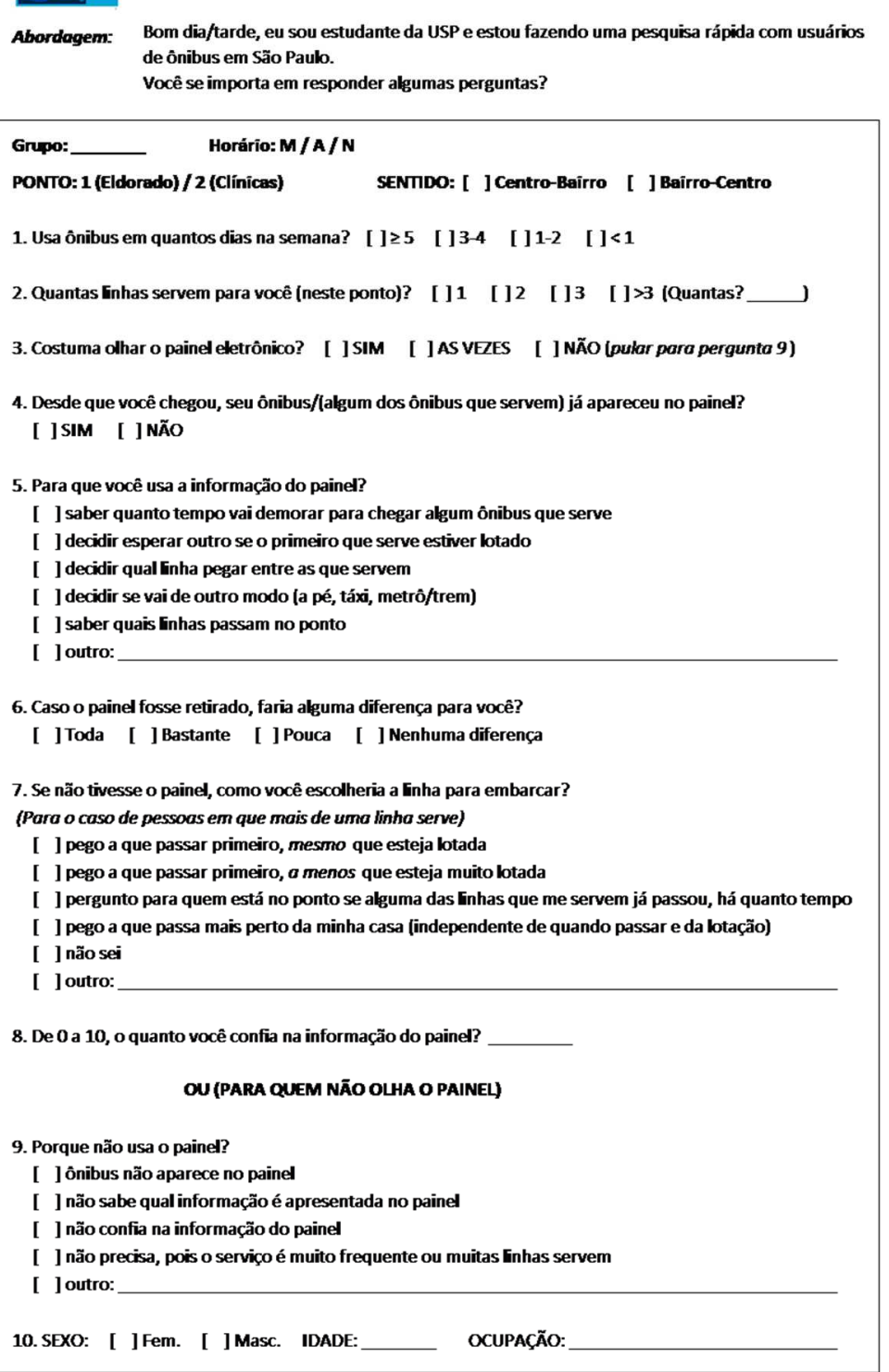

Figura 1. Formulário utilizado para as entrevistas nas paradas de ônibus

Para ambos os pontos, os dados relativos à operação do sistema de ônibus e às informações disponibilizadas ao usuário foram coletados através da filmagem do painel e do registro simultâneo do horário da chegada dos veículos, de forma a permitir a comparação entre a previsão e a chegada real. Já os dados referentes ao uso do painel pelo usuário e à sua percepção da qualidade da informação foram coletados por entrevistas realizadas nos dois sentidos de cada ponto, seguindo o questionário da Figura 1. As questões 5, 7 e 9 poderiam ter mais de uma alternativa assinaladas e o entrevistador foi instruído a não ler as alternativas para o entrevistado, e sim extrair o conteúdo a partir de sua resposta.

Quanto às entrevistas, o total foi de 329 questionários válidos. No que diz respeito aos dados do sistema, foram coletados mais de 4100 previsões de chegada de ônibus apresentadas nos painéis e cerca de 500 registros de chegada de ônibus (em ambos os casos com precisão de minuto) para serem comparados com suas respectivas previsões. Cabe notar que, para previsões de chegada inferiores a cerca de dois minutos, o painel deixa de informar o tempo previsto e passa a apresentar a mensagem
"Aprox", em referência ao fato de que o veículo está se aproximando do ponto de parada. Cerca de metade das observações de previsão de chegada coletadas enquadra-se nesta categoria. As várias previsões registradas para um mesmo ônibus (à medida que este se aproxima do ponto de parada) foram consideradas como observações independentes, em função das características do algoritmo de previsão utilizado pela SPTrans. A abrangência final de locais, sentidos e horários pesquisados é apresentada na Tabela 1.

\section{RESULTADOS}

Em concordância com os objetivos da pesquisa, descritos no item 1, os resultados são apresentados sob duas perspectivas: qualidade objetiva e percebida da informação divulgada pelos PMV (objeto do item 3.1) e caracterização do uso dos painéis por parte dos entrevistados (objeto do item 3.2). 
LAVIERI, P.S.; STRAMBI, O.; ARBEX, R.; CARVALHO, E.C.S.

Tabela 1. Abrangência da coleta de dados

\begin{tabular}{llllll}
\hline & \multicolumn{5}{c}{ Tabela 1. Abrangência da coleta de dados } \\
& Ponto & Sentido & Horário & $\begin{array}{l}\text { Dados do } \\
\text { sistema }\end{array}$ & Entrevistas \\
\hline 1 & Clínicas & Bairro & Manhã & Não & Sim \\
2 & Clínicas & Bairro & Almoço & Sim & Sim \\
3 & Clínicas & Bairro & Tarde & Não & Sim \\
4 & Clínicas & Centro & Manhã & Sim & Sim \\
5 & Clínicas & Centro & Almoço & Sim & Sim \\
6 & Clínicas & Centro & Tarde & Sim & Não \\
7 & Eldorado & Centro & Manhã & Sim & Sim \\
8 & Eldorado & Centro & Almoço & Não & Sim \\
9 & Eldorado & Bairro & Manhã & Sim & Não \\
10 & Eldorado & Bairro & Tarde & Sim & Sim \\
11 & Eldorado & Bairro & Almoço & Não & Sim \\
\hline
\end{tabular}

\subsection{Qualidade da informação}

A partir dos dados coletados em campo, foi possível classificar cada previsão fornecida por um dado painel (correspondente a uma determinada combinação de ponto de parada, sentido e horário, conforme a Tabela 1) quanto à sua acurácia, bem como classificar a combinação ponto/sentido/horário quanto à acurácia do conjunto das suas informações. Trata-se, portanto, de uma avaliação objetiva da qualidade. $\mathrm{O}$ critério adotado para classificar uma informação como "boa" ou "ruim" leva em conta a comparação entre o registro da previsão do painel e o registro do momento em que se observa a chegada efetiva do veículo ao ponto, conforme descrição a seguir:

Para as situações em que o painel fornecia os horários previstos de chegada dos veículos:

- veículo chegando ao ponto antes do horário previsto pelo painel > informação qualificada como "ruim";

- veículo chegando ao ponto 4 ou mais minutos depois do horário previsto pelo painel > informação qualificada como "ruim";

- veículo chegando ao ponto, no máximo, 3 minutos depois do horário previsto pelo painel > informação qualificada como "boa".

Para as situações em que o painel informava que o veículo estava se aproximando do ponto (identificadas pela mensagem "Aprox"):

- veículo chegando ao ponto 4 ou mais minutos depois da indicação "Aprox" aparecer no painel > informação qualificada como "ruim";

- veículo chegando ao ponto, no máximo, 3 minutos depois da indicação "Aprox" aparecer no painel > informação qualificada como "boa".

A Tabela 2, a seguir, mostra as estatísticas (percentual de informações boas e ruins) para cada combinação ponto/sentido/horário. Em geral, de $60 \%$ a $80 \%$ das previsões podem ser consideradas boas, segundo os critérios acima. Como o painel exibe informações sobre os oito veículos mais próximos do ponto, é esperado que a proporção de mensagens "Aprox" seja maior quanto maior a frequência de ônibus no ponto. Não foi possível estabelecer uma correlação entre a frequência e a qualidade da informação.

Esses resultados podem ser comparados com os obtidos em pesquisa realizada pela própria SPTrans. Ferreira (2012) apresenta os seguintes resultados:

- $67 \%$ de acerto no minuto exato da previsão;

- $90 \%$ de acerto com erro de 2 minutos (para mais ou para menos);

- $92 \%$ de acerto com erro de 3 minutos (para mais ou para menos); e

- $96 \%$ de acerto com erro de 5 minutos (para mais ou para menos).

Duas diferenças importantes devem ser destacadas nesta comparação. Primeiro, estas previsões referem-se ao sistema Olho Vivo, disponibilizado na internet pela SPTrans, que utiliza uma versão melhorada do algoritmo de previsão em relação àquela utilizada para os PMV. Segundo, os casos de adiantamento do veículo em relação à previsão são tratados da mesma maneira que os casos de atraso. Porém, do ponto de vista do usuário, o primeiro tipo de erro é muito mais grave do que o segundo, uma vez que um erro de adiantamento pode induzir o usuário a esperar por um ônibus que efetivamente já passou. A diferença nos critérios contribui para a diferença entre a qualidade medida pelos dois estudos, $92 \%$ de acerto contra $68 \%$ de acerto (considerando erros de até 3 minutos em ambos os casos).

A confiança do usuário em relação à informação fornecida também pode ser afetada no caso de veículos que atendam ao ponto de parada não terem suas previsões apresentadas no painel. Este tipo de erro pode ocorrer

Tabela 2. Qualidade das informações de previsão de chegada dos veículos

\begin{tabular}{|c|c|c|c|c|}
\hline $\begin{array}{l}\text { Pon- } \\
\text { to/Sentido/Horár } \\
\text { io } \\
\text { (cf. Tabela 1) }\end{array}$ & $\begin{array}{l}\text { \% de registros } \\
\text { indicando } \\
\text { "Aprox" }\end{array}$ & $\begin{array}{l}\text { Frequência de } \\
\text { onibus no ponto } \\
\text { em } 1 \text { hora }\end{array}$ & $\begin{array}{l}\text { \% de informação } \\
\text { Class. Ruim }\end{array}$ & $\begin{array}{l}\text { \% de informação } \\
\text { Class. Boa }\end{array}$ \\
\hline 2 & $29,0 \%$ & 55 & $31,0 \%$ & $69,0 \%$ \\
\hline 4 & $65,5 \%$ & 127 & $21,5 \%$ & $78,5 \%$ \\
\hline 5 & $36,9 \%$ & 83 & $40,6 \%$ & $59,4 \%$ \\
\hline 6 & $38,0 \%$ & 58 & $39,0 \%$ & $61,0 \%$ \\
\hline 7 & $48,2 \%$ & 121 & $27,1 \%$ & $72,9 \%$ \\
\hline 9 & $42,5 \%$ & 94 & $27,5 \%$ & $72,5 \%$ \\
\hline 10 & $64,5 \%$ & 130 & $37,3 \%$ & $62,7 \%$ \\
\hline
\end{tabular}




\section{Proporção das Notas de Confiança no Painel}

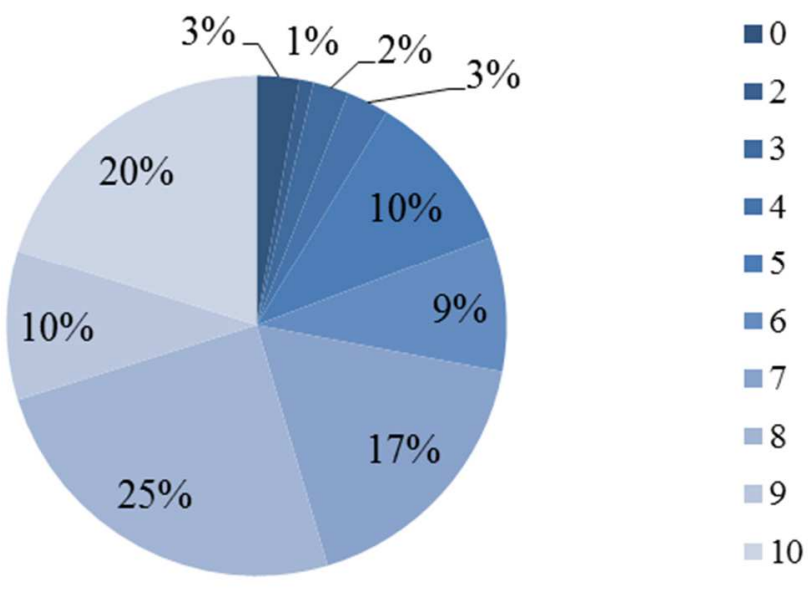

Figura 2. Distribuição das notas de confiança no painel dadas pelos entrevistados

devido a uma desatualização do sistema com relação à representação das linhas ou porque os dados enviados pelo veículo para o sistema foram descartados per critérios de filtragem de dados do algoritmo (Ferreira, 2012). Do total de registros de chegada de veículos observados neste estudo (cerca de 500), $16 \%$ não possuía previsão correspondente no PMV. Esta porcentagem não apresentou grande variação entre os pontos, sentidos e horários.

A percepção dos entrevistados quanto à qualidade (subjetiva) da informação foi capturada pela questão número oito do questionário de pesquisa e é representada pela nota que o entrevistado atribuiu à sua confiança no painel. Conforme a Figura 2, verifica-se que mais de $90 \%$ dos usuários atribuíram nota 5 ou superior, com $55 \%$ atribuindo notas entre 8 e 10. A média foi de 7,33 (desvio padrão de 2,25). Não foi observada a nota 1 nas respostas. Vale ressaltar que a amostra das notas de confiabilidade é restrita a $65 \%$ do total de entrevistados (que corresponde aos usuários que declararam utilizar o painel). Embora investigada, não foi encontrada nenhuma correlação evidente entre as notas atribuídas pelos usuários e as classificações da qualidade objetiva da informação correspondentes ao ponto, sentido e horário em que foram entrevistados. Este resultado era esperado, uma vez que as notas atribuídas pelos indivíduos são formadas com base em experiências anteriores e, provavelmente, incluem viagens realizadas a partir de outros pontos, sentidos e horários.

\subsection{Caracterização do uso dos painéis}

Antes de explorar os elementos determinantes para o uso (ou o não uso) dos painéis, através da estimação de modelos de escolha discreta do tipo logit binomial (item 3.2.2), cabe realizar a caracterização socioeconômica e relativa aos hábitos de uso do ônibus e dos painéis por parte dos entrevistados.

\subsubsection{Caracterização da amostra e do comportamento de uso do painel}

Quanto às características socioeconômicas, a amostra de entrevistados possui diversidade nas idades e ocupações. Verifica-se que as ocupações dos entrevistados estão mais ligadas ao setor de serviços e, em geral, não exigem qualificação especializada ou ensino superior. Estudantes representam 18,2\% da amostra. A maior concentração de entrevistados por idade está na faixa de 21-40 anos (46\%), assim como na faixa de 41-60 anos (28\%). Apenas 10\% possuem mais que 61 anos e $13 \%$ menos que 20 anos. São mulheres $61 \%$ da amostra, contra $39 \%$ de homens.

Quanto à frequência de uso do ônibus durante a semana, a maior parte dos entrevistados disse que utiliza diariamente $(59 \%)$, enquanto $14 \%$ usam de três a quatro vezes por semana, $12 \%$ de uma a duas vezes, e $15 \%$ usam menos de uma vez por semana.

A quantidade de linhas que servem a um usuário para a realização da sua viagem varia de acordo com o sentido, como apresentado na Figura 3. Nas viagens sentido centro, a maior parte poderia ser realizada com quatro ou mais linhas (44\%) e cerca de um quarto (26\%) poderia ser realizada por apenas uma linha. Já no sentido bairro, pelo fato de os destinos serem mais dispersos espacialmente, uma parcela considerável dos entrevistados podia embarcar apenas em uma única linha (38\%), enquanto somente $19 \%$ podia utilizar quatro ou mais linhas.

A questão 5 do questionário tinha o objetivo de identificar para qual finalidade o entrevistado utilizava a informação fornecida pelo painel. Porém, durante a análise de dados e ao se conversar com os entrevistadores, foi possível identificar um problema ocorrido durante a apresentação desta questão aos usuários, o que acarretou em um viés nas repostas. Verificou-se que a primeira alternativa listada no questionário englobava as três alternativas seguintes, uma vez que para decidir esperar outro ônibus ou decidir em qual linha embarcar, ou ainda mudar de modo, é preciso saber quanto tempo vão demorar os ônibus. Em princípio este aspecto não seria um problema, pois o entrevistador poderia simplesmente marcar duas ou mais alternativas. Porém, em muitos casos, o entrevistado não sabia o que responder e, para ajuda-lo, o entrevistador lia a primeira alternativa ("para saber quanto tempo vai demorar para chegar algum ônibus que serve") e, caso o usuário a apontasse como verdadeira para si, o entrevistador não apresentava as demais, ou seja, a resposta era induzida e limitada à primeira alternativa, gerando um viés nos resultados desta questão.

Quanto ao impacto para o usuário de uma possível retirada do painel, as respostas apresentam uma distribuição equilibrada: $55 \%$ responderam "bastante" ou "toda" e $45 \%$ responderam "pouca" ou "nenhuma" diferença. Além disso, ao se relacionar estas respostas com a frequência de 


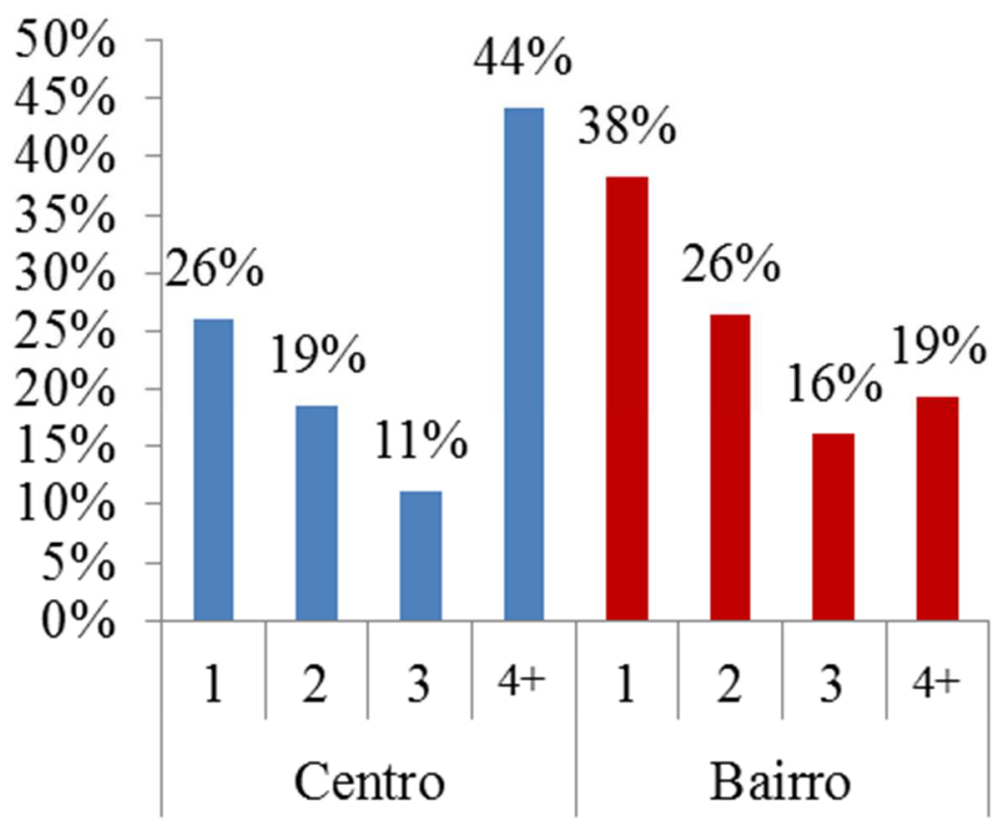

Figura 3. Número de linhas que servem para realização da viagem

utilização do ônibus ou com a quantidade de linhas que serviam ao entrevistado, não se identificou nenhuma correlação.

Já a informação de como o usuário escolheria a linha para embarcar caso não houvesse o painel foi comparada com a quantidade de linhas que serviam para sua viagem. Observou-se que, sem informação sobre a chegada dos próximos veículos, apenas quando quatro ou mais linhas servem, a proporção de usuários que rejeita um ônibus lotado supera a dos que pegam o primeiro a passar, mesmo lotado, como ilustrado na Figura 4. Observando os respondentes que disseram, na questão 5, utilizar a informação do painel para decidir se esperariam um próximo onibus caso o seu ônibus de maior interesse chegasse lotado, identificou-se o mesmo padrão. Mesmo com a informação do painel, o usuário decide esperar por um próximo ônibus apenas se quatro ou mais linhas lhe servirem. Este resultado parece indicar que a informação ao usuário não é determinante para sua decisão de esperar por um próximo ônibus; é necessário que muitas linhas o atendam.

Por fim, ao se analisar as justificativas do não uso do painel, a resposta mais frequente foi o fato do serviço ser muito frequente, o que é coerente com o fato de que a maioria dos entrevistados que deu esta justificativa podia escolher entre 3 ou mais linhas para sua viagem.

\subsubsection{Fatores determinantes do uso do painel}

Dentre várias especificações estimadas, o modelo logit binomial exibido na Tabela 3 foi considerado o mais adequado para retratar o comportamento da amostra quanto ao uso (ou não) do PMV. Verifica-se que todos os parâmetros estimados são diferentes de zero ao nível de significância de $5 \%$, e o teste da razão da máxima veros-

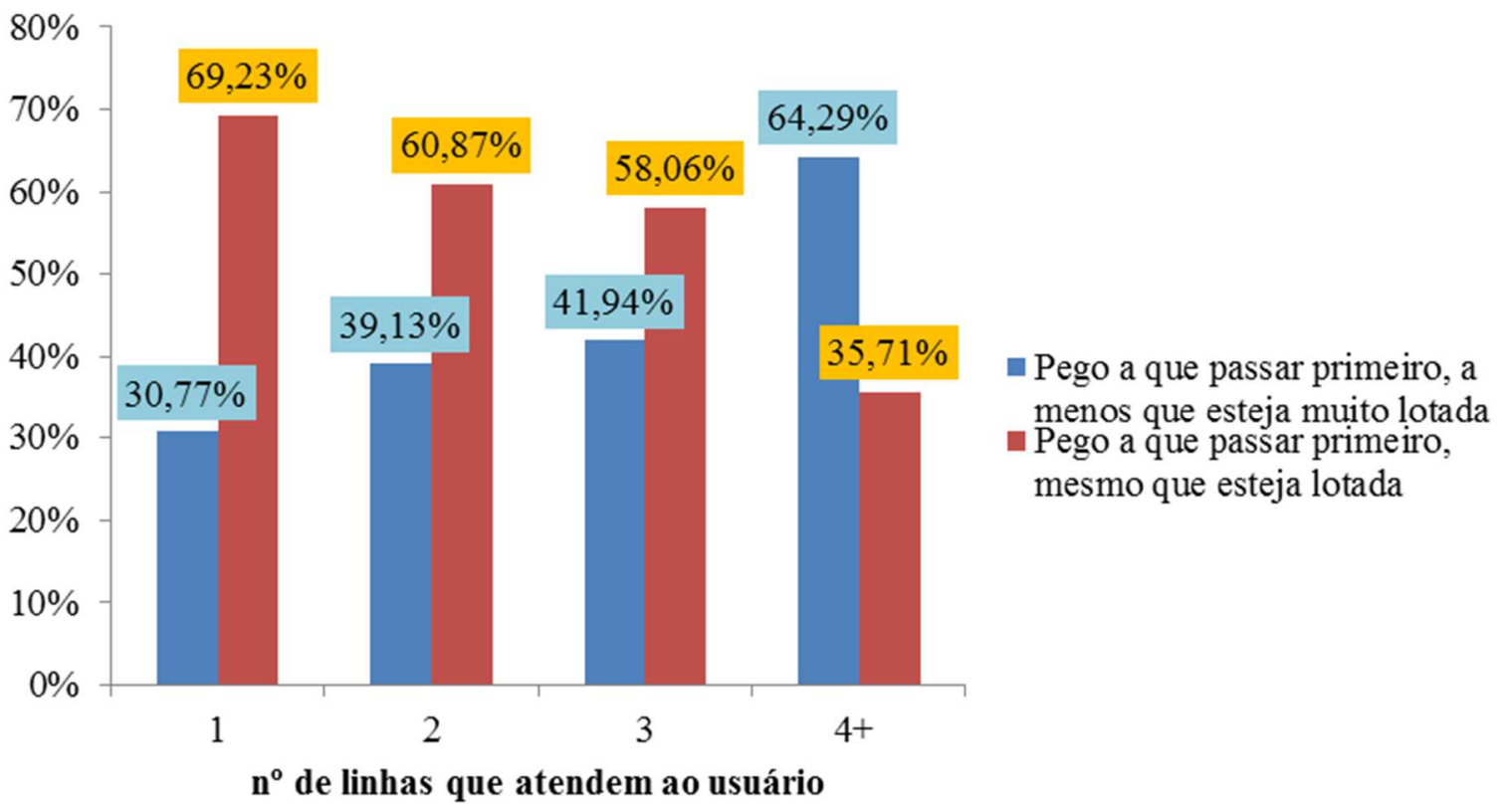

Figura 4. Comportamento diante da lotação do ônibus em função do número de linhas que servem, na ausência do painel 


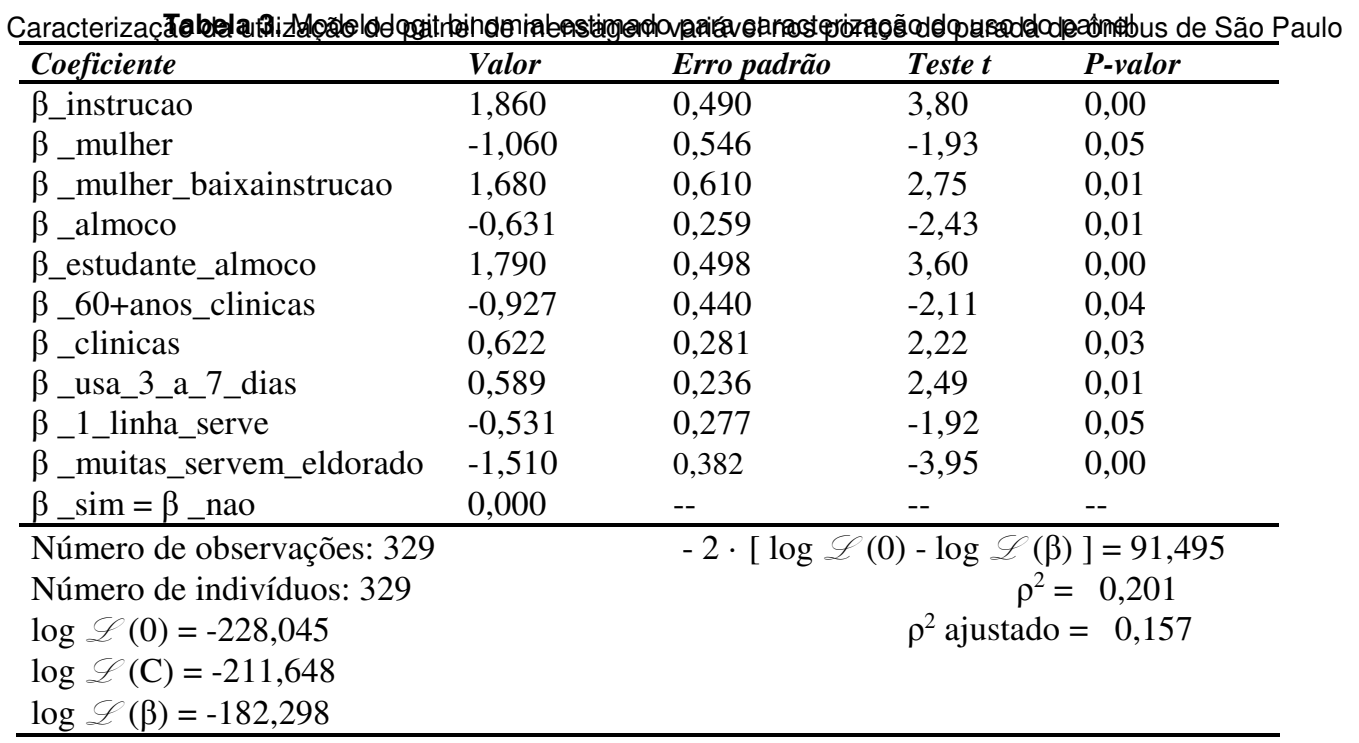

similhança rejeita a hipótese de que os parâmetros sejam iguais a zero. $\beta \_$sim e $\beta \_$nao, que são as constantes das alternativas usa painel e não usa painel (respectivamente), foram fixadas em zero neste modelo, visto que mostraramse não significativas em estimações anteriores desta mesma especificação. Cabe salientar que a alternativa usa painel refere-se ao comportamento dos 216 entrevistados que responderam "sim" e "às vezes" na terceira questão do formulário exibido na Figura 1 ("Costuma olhar o painel eletrônico?"), enquanto a alternativa não usa painel referese, obviamente, ao comportamento dos 113 entrevistados que responderam "não" nesta mesma questão.

Todos os parâmetros, com exceção de $\beta \_$nao, fazem parte da função utilidade da alternativa usa painel. Com exceção das constantes das alternativas ( $\beta \_$sim e $\beta \_$nao), todos os coeficientes do modelo são multiplicados por variáveis dummy, que representam características socioeconômicas, variáveis relativas à frequência de uso de ônibus e relativas à viagem que o usuário faria momentos depois de ser entrevistado. Embora tal viagem pudesse apenas pontualmente influenciar os hábitos de uso do painel (por ser pouco frequente, por exemplo), assumiu-se que era uma viagem representativa das viagens normalmente feitas pelo entrevistado e capaz de influenciar seus hábitos de uso do painel, a fim de permitir a interpretação do modelo estimado. A seguir são analisados os resultados, partindo da observação da Tabela 3 .

No que diz respeito ao grau de instrução (inferido a partir da ocupação), verifica-se que indivíduos com mais instrução (a partir do nível técnico), tendem a utilizar mais o painel do que usuários menos instruídos, sendo que o efeito da instrução é bastante acentuado para homens, e suave para mulheres (mostrando que as entrevistadas, instruídas ou não, tendem a usar o painel). Acredita-se que, quanto maior a instrução, maior a facilidade de uso e familiaridade com tecnologias como o painel.

Observa-se que, no horário do almoço, existe tendência a não usar o painel. Pode-se supor que nesta faixa horária haja menor concentração de entrevistados viajando por motivo de trabalho, e, portanto, com menor pressão de tempo (ao contrário do que pode ocorrer, por exemplo, no período da manhã) e decorrente menor necessidade de uso do painel. Adicionalmente, entre os entrevistados no horário do almoço, há maior proporção de usuários pouco frequentes de ônibus do que nos demais horários (34\% contra 20\%). Há, no entanto, uma exceção: estudantes que viajam no horário do almoço tendem a utilizar o painel (sendo que estudantes do sexo masculino apresentam maior tendência ao uso do painel do que os do sexo feminino). Supõe-se que parte dos estudantes viajando no horário do almoço tenha o estudo como motivo de viagem, e sofram, portanto, algum grau de pressão de tempo (o que os motivaria a usar o painel).

Verifica-se que os entrevistados do ponto Clínicas apresentam maior tendência ao uso do painel do que os entrevistados do ponto Eldorado, o que pode ser atribuído ao fato de que a frequência de ônibus é menor no ponto Clínicas (o que geraria maior necessidade de informação a respeito da chegada dos veículos). No entanto, pode-se notar que entrevistados a partir de 60 anos, mesmo estando no ponto Clínicas, tendem a não utilizar o painel. Acredita-se que parte destes entrevistados possa ter algum tipo de dificuldade de visão ou mesmo de interpretação dos dados do PMV, dificultando seu uso. Ressalta-se que o ponto Clínicas está situado próximo ao Hospital das Clínicas em São Paulo, sendo frequentado também por pacientes da instituição.

Conforme o esperado, a frequência de uso de ônibus tem impacto positivo na utilização do painel: entrevistados que usam ônibus três ou mais dias na semana apresentam maior tendência ao uso do painel.

No que diz respeito à quantidade de linhas que servem para a viagem do entrevistado, verifica-se que, quando há apenas uma opção para o destino desejado, há tendência de não usar o painel. Tal fato é condizente com a menor serventia do painel para esta situação: ele ajudará somente informando o horário de chegada do veículo, mas não será útil para decisões de maior complexidade, como a escolha de uma linha dentre várias opções. Aparentemente os passageiros preferem, para o caso de apenas uma linha disponível, monitorar a chegada do veículo na própria via. Outra situação que ocasiona tendência de não uso do painel é a existência de muitas linhas (quatro ou mais) disponíveis para o destino desejado, que passem por um ponto com grande fluxo de veículos (como é o caso do ponto Eldorado). Ou seja: além de o usuário poder embarcar em várias linhas diferentes, há grande probabilidade de que não demorem a chegar ao ponto. Para este caso, também, parece ser mais vantajoso para o passageiro monitorar a chegada dos veículos na própria via. 


\section{CONCLUSÕES}

$\mathrm{O}$ artigo analisou a qualidade e o uso feito por parte dos usuários das informações sobre previsões da chegada de veículos em pontos de ônibus, disponibilizadas nas paradas através de painéis de mensagem variável. Os dados utilizados foram coletados em duas paradas no corredor Campo Limpo/Rebouças/Centro em São Paulo, nos dois sentidos e em diferentes períodos do dia. Foram registradas as previsões mostradas nos painéis e as chegadas dos veículos aos pontos, e realizadas entrevistas com os usuários sobre o uso dos painéis.

Os resultados indicam que o sistema apresenta uma proporção de previsões consideradas boas em $68 \%$ dos casos, com atrasos de 3 minutos ou menos em relação ao horário previsto de chegada dos veículos aos pontos. A percepção dos usuários com relação à qualidade da informação disponibilizada está alinhada com este resultado: cerca de 2/3 dos usuários declara utilizar a informação fornecida, para diferentes finalidades e, entre estes, a média das notas atribuídas à confiança no painel é 7,33, com $55 \%$ atribuindo notas entre 8 e 10 .

A possível retirada do painel seria vista como negativa por $55 \%$ dos entrevistados que declararam usar o painel. O não uso do painel tende a ocorrer em maior proporção entre os usuários que dispõem de muitas linhas para realizar sua viagem.

Para identificar os efeitos das principais características socioeconômicas e hábitos de utilização dos ônibus sobre o uso da informação dos painéis, foi estimado um modelo logit binomial tendo como variável dependente o uso ou não do painel. Através da sua análise, verificou-se que:

- maiores níveis de instrução afetam positivamente o uso dos painéis por parte dos entrevistados (e de maneira mais intensa para homens do que para mulheres);

- no horário do almoço há menor tendência a usar os painéis, em relação aos horários de pico da manhã e da tarde, o que possivelmente está associado a uma menor pressão de tempo (sendo que os estudantes não seguem esta tendência, usando o painel no horário do almoço);

- o uso do painel está associado à menor frequência de veículos passando pelo ponto (sendo que usuários a partir de 60 anos não seguem esta tendência, não usando o painel mesmo em pontos de menor frequência de veículos);

- o uso mais frequente de ônibus tem impacto positivo na utilização do painel;

- quando há apenas uma opção de linha para o destino desejado, há tendência de não usar o painel, bem como quando há muitas linhas (quatro ou mais) disponíveis para o destino, passando por um ponto com grande fluxo de veículos.

Os resultados desta pesquisa demonstram a utilidade da disponibilização de informação aos usuários do transporte público, em particular as previsões de chegada dos veículos aos pontos. A implantação e ampliação desses sistemas devem ser consideradas pelos órgãos responsáveis pela gestão do transporte público, em virtude dos benefícios percebidos pelos usuários. Em um horizonte de longo prazo, porém, esta análise deve considerar a crescente penetração de smartphones ou outros dispositivos móveis, com capacidade de obter este tipo de informação através de consultas à internet.

\section{AGRADECIMENTOS}

Os autores agradecem aos alunos de graduação da Escola Politécnica da Universidade de São Paulo, da disciplina PTR2577 do $1^{\circ}$ semestre de 2013 pela participação no planejamento e realização da coleta de dados. Agradecem ainda à CAPES e ao CNPq pelo apoio à pesquisa.

\section{REFERÊNCIAS}

Ben-Elia, E.; Di Pace, R.; Bifulco, G. N.; Shiftan, Y. (2013) The impact of travel information's accuracy on route-choice. Transportation Research Part C, v. 26, p. 146-159.

Bierlaire, M. (2003) Biogeme: a free package for the estimation of discrete choice models. In: 3rd SWISS Transportation Research Conference. Conference Paper. Ascona, 2003.

Bonsall, P. Traveller behavior: decision-making in an unpredictable world. Journal of Intelligent Transportation Systems, v. 8, n. 1, p. $45-60$.

Chorus, C. G.; Arentze, T. A.; Molin, E. J. E. (2006a) The value of travel information: decision strategy-specific conceptualizations and numerical examples. Transportation Research Part B, v. 40, n. 6, p. 504-519. DOI: 10.1016/j.trb.2005.08.002

Chorus, C. G.; Molin, E. J. E.; Van Wee, B. (2006b) Use and effects of advanced traveller information services (ATIS): a review of the literature. Transport Reviews, v. 26, n. 2, p. 127149. DOI: $10.1080 / 01441640500333677$

Dziekan, K.; Kottenhoff, K. (2007) Dynamic at-stop real-time information displays for public transport: effects on customers. Transportation Research Part A, v. 41, n. 6, p. 489-501. DOI: 10.1016/j.tra.2006.11.006

Ettema, D.; Timmermans, H. (2006) Costs of travel time uncertainty and benefits of travel time information: conceptual model and numerical examples. Transportation Research Part C, v. 14, n. 5, p. 335-350. DOI: $10.1016 /$ j.trc.2006.09.001

Ferreira, M. L. (2012) Olho Vivo - Sistema de informação online ao usuário do transporte coletivo na cidade de São Paulo. Coletivo - Revista Técnica da SPTrans, n. 1, p.120-145.

Larsen, O. I.; Sunde, Ø. (2008) Waiting time and the role and value of information in scheduled transport. Research in Transportation Economics, v. 23, n. 1, p. 41-52. DOI: 10.1016/ j.retrec.2008.10.005

TRB (2003) Real-time Bus Arrival Information Systems: A Synthesis of Transport Practice. Transportation Research Board, Washington D.C..

Whately, I. M. (2012) O Papel dos Ônibus nos Transportes Públicos de São Paulo. Coletivo - Revista Técnica da SPTrans n. 1, p.18-33. 